\title{
ON CENTRAL TOPOLOGICAL GROUPS
}

\author{
BY \\ SIEGFRIED GROSSER( $\left.{ }^{1}\right)$ AND MARTIN MOSKOWITZ( $\left.{ }^{2}\right)$
}

1. Introduction. Let $G$ be a locally compact topological group and $Z(G)$ its center. We shall be concerned with the class $[Z]$ of all locally compact groups $G$ such that $G / Z(G)$ is compact. It turns out that the various structural results on groups in $[Z]$ generalize and unify in a natural manner those of compact groups on the one hand, and of locally compact abelian groups on the other. The present paper gives complete proofs of the results announced in Bull. Amer. Math. Soc. 72 (1966), 831-836 under the same title.

$\$ 2$ contains various elementary conditions for groups $G$ to be in $[Z]$ and [SIN] and is preparatory in character.

Theorems 3.1 and 3.2 of $\S 3$ are extension theorems for certain continuous homomorphisms $\phi: N \rightarrow V$, where $N$ is a closed normal subgroup of $G, G \in[Z]$, and $V$ is a vector group. An essential fact which we utilize is contained in Lemma 1, due to P. Cartier ([1, p. 22-01] or [5, p. 36]). Various conditions are imposed upon $N$ (or $Z(G)$ ) and upon $\phi$; the extension $\psi: G \rightarrow V$ of $\phi$ will be either a crossed homomorphism with respect to a given representation $\rho$ of $G$ on $V$ or a homomorphism (if no representation is involved). We show by an example that, in contrast to abelian group theory, torus-valued homomorphisms do not extend. An application of one of these extension theorems gives a direct estimate of the rank of the fundamental group of an analytic group in $[Z]$. By sharpening the estimate given by P. Smith in [18, pp. 210-229], we answer a question raised there.

In order to facilitate the exposition we introduce the following terminology. We denote by $\mathfrak{A}$ (or $\mathfrak{A}(G)$ ) the group of topological group automorphisms of the locally compact group $G$. (As is well known, in the compact-open topology, $\mathfrak{A}$ is not-in general-a topological group; a finer topology must be employed.) The group $\Im$ of inner automorphisms of $G$ is a normal (but not necessarily closed) subgroup of $\mathfrak{A}$. If $\mathfrak{B}$ is any subgroup of $\mathfrak{A}$ we say that $G$ has small $\mathfrak{B}$-invariant neighborhoods of the identity if every neighborhood of the identity in $G$ contains a $\mathfrak{B}$-invariant neighborhood of the identity. In particular, if $\mathfrak{B}=\mathfrak{F}$, we simply say that $G$ has small invariant neighborhoods of the identity. The class of all such groups will be denoted by [SIN]. Groups of this sort were first studied by G. Mostow in [13] and by K. Iwasawa in [7] $\left({ }^{3}\right)$.

Received by the editors October 11, 1965.

( $\left.{ }^{1}\right)$ Research partially supported by National Science Foundation GP-1610.

${ }^{(2)}$ Research partially supported by National Science Foundation and U.S. Army Research Office, Durham.

${ }^{(3)}$ For a survey of the pertinent literature as well as additional results see K. H. Hofmann and P. S. Mostert [6]. 
In $\S 4$, which contains the basic structure theorems, we first study the relationship between $[Z]$-groups and $[S I N]$-groups. To effect this, we prove an Ascoli Theorem for subgroups $\mathfrak{B}$ of the automorphism group $\mathfrak{A}(G)$, for a general locally compact group $G$. (We note in this connection that here the general Ascoli Theorem is not applicable.) This theorem states that $\overline{\mathfrak{B}}$ is compact if and only if $G$ has small $\mathfrak{B}$-invariant neighborhoods of the identity and the $\mathfrak{B}$-orbits of points of $G$ have compact closure. The conclusion points up the relevance of the generalization introduced above of the notion of small invariant neighborhoods of the identity. Closely related with $[Z]$ is the class $[F I A]^{-}$of locally compact groups $G$ with the property that $\bar{\Im}$ is compact; it was introduced by R. Godement $\left({ }^{4}\right)$. Theorem 4.2 characterizes these groups and places them between $[Z]$ and [SIN]. These facts together with Theorem 4.3 yield the result that for connected (locally compact) groups these notions coincide.

Next we prove the structure theorem (Theorem 4.4) for groups in [Z]. If $G \in[Z]$ then $G=W \times H$ (direct product), where $W$ is a vector group and $H$ contains $K$, a compact, open, normal subgroup. This theorem generalizes substantially the classical structure theorem for locally compact abelian groups $[19$, p. 110]. In [7, pp. 345-348] K. Iwasawa studied locally compact groups $G$ whose commutator subgroup has compact closure. We denote this class by $[F D]^{-}$. An application of the structure theorem yields the fact that groups in $[Z]$ satisfy this condition. (This generalizes a well-known theorem of discrete group theory [14].) Moreover, any group in $[Z]$ is a projective limit of Lie groups (Theorem 4.5).

Utilizing the results quoted as well as [7] and a counterexample due to G. D. Mostow [13] we conclude the section with an investigation of the relationships between the four classes of groups which have arisen. The results of $\S 4$ together with [7] and [13] may be regarded as the clarification called for by A. Weil of the complex of questions raised on p. 129 of [19].

It is perhaps in order here to make a remark on the history of these questions. In principle, they concerned (a) a structure theorem for connected [SIN]-groups or connected maximally almost periodic groups, respectively, (b) a generalization of the structure theorem for locally compact abelian groups, and (c) the problem of approximation by Lie groups. Weil listed the fact that a statement of the theorem quoted in (a), for second countable groups, had been given by L. Pontrjagin, whereas a proof (in this case) had been given by H. Freudenthal. As regards (b), the author listed van Kampen's assertion that the theorem in question generalized to [SIN]-groups. He added, however, referring to both (a) and (b) that "il serait désirable que la question fût reprise et definitivement élucidée; même avec les indications données par ces auteurs, il n'est pas certain que toute difficulté en soit déjà éliminée."

With the appearance of the papers by G. D. Mostow and K. Iwasawa quoted

$\left(^{4}\right)$ This author stated a number of results on $[F I A]^{-}$-groups [3], which were later withdrawn [4]. 
above (which, among other things, corrected an erroneous assertion made by Weil) as well as of a paper by Kuranishi and an Amer. Math. Soc. Memoir on splitting in topological groups by K. H. Hofmann and P. S. Mostert [6], some of the questions raised above were clarified. To our knowledge, Hofmann and Mostert were the first to study the subject systematically. In the pertinent chapter of [6] they also list an attempt made by J. Braconnier to solve problem (b). In criticism of their approach, however, it must be said that because of an example given by Braconnier (J. Math. Pures Appl. 27 (1948), 1-85), there seems to be a serious problem regarding the advisability of their use of the compact-open topology.

We now continue the outline of the present paper.

$\S 5$ gives the finer structural properties of groups in $[Z]$. The proofs depend mainly on the results of $\S 4$ in combination with other known facts. As we noted earlier, they generalize and unify known facts about abelian groups and compact groups. Theorem 5.1 says that the set of points that lie on one-parameter subgroups of a connected group in $[Z]$ is dense in the group. Then we prove that normal vector subgroups of groups in $[Z]$ are direct factors. Theorem 5.3 concerns a problem in group extensions.

We then define periodic elements of $G$ to be elements which generate compact subgroups of $G$. If $G \in[Z]$ then the set of these elements, $P$, forms a characteristic subgroup, and $G / P=W \times D$ (direct product), where $W$ is a vector group and $D$ is a discrete, torsion free abelian group (Theorem 5.4). The real-valued continuous homomorphisms separate the points of $G$ if and only if $G=W \times D$. If $P$ is compact, it is the maximum compact subgroup of $G$. Conversely, if $G$ possesses a maximum compact subgroup, that subgroup must be $P$. Theorem 5.5 gives a necessary and sufficient condition on $G$ for this to be the case. It follows that every compactly generated group $G$ in $[Z]$ possesses a maximum compact subgroup and that every such $G$ possesses a central lattice (Corollaries 1,2 ). Next, we give an example to show that, in contrast to abelian group theory, the extension (1) $\rightarrow P \rightarrow G \rightarrow$ $G / P \rightarrow(1)$ obtained in Corollary 1 does not split.

In discrete group theory, the concept of division closure of subgroups generalizes that of torsion subgroup. Likewise, the concept of periodic subgroup can be generalized. Thus we give the definition of the division closure (in the topological sense) of a subgroup of a locally compact group. This, in turn, generalizes the notion of division closure for discrete groups. For groups in $[Z]$, this closure operation, performed on subgroups, yields closed, normal, division-closed subgroups and the expected relationship between the division closure and the periodic subgroup holds (Theorem 5.6). We conclude with some results of a lattice theoretic nature.

Finally, a word about terminology. It seems unlikely that the bulk of the results stated above (in particular, the structure theorem) extend to $[F I A]^{-}$, i.e., to the class of groups called "groupes centraux" by R. Godement. Moreover, as will be shown in a subsequent publication, the same distinction prevails with respect to representation theory. For these reasons we feel justified in our use of the term "central." 
Much of our notation is in conformity with B. H. Neumann who introduced notation for a number of "finiteness conditions" in discrete group theory [15].

In conclusion, we express warmest thanks to Professor G. P. Hochschild for his careful criticism of the results and his generous help in the preparation of this paper.

\section{Preparatory theorems.}

THEOREM 2.1. (1) Let $G \in[Z]$ and let $H$ be a closed subgroup of $G$. Then $H \in[Z]$ if (a) $H$ is open in $G$, or (b) $H$ is $\sigma$-compact and $H Z$ is closed, or (c) $Z$ is open in $G\left(^{5}\right)$. (2) Let $G \in[Z]$ and $H$ be a continuous homomorphic image of $G$. If $H$ is locally compact then $H \in[Z]$.

(3) If $G \in[Z]$ and $G$ is a direct product of a family of groups $\left\{G_{\alpha}\right\}$ then each $G_{\alpha} \in[Z]$. Conversely, if each $G_{\alpha} \in[Z]$ and $G$ is locally compact, then $G \in[Z]$.

(4) Let $G$ and $H$ be locally compact connected groups and $f: G \rightarrow H$ be a continuous open epimorphism with discrete kernel. If $H \in[Z]$ then $G \in[Z]$.

Proof. (1) In case (a) the correctness of the assertion follows from the Second Isomorphism Theorem; in (b) from the same theorem and the Open Mapping Theorem [17, p. 132]. In (c) one sees easily that $Z(H)$ is of finite index in $H$.

(2) Let $f: G \rightarrow H$ be a continuous epimorphism. Evidently, $f(Z(G)) \subseteq Z(H)$, so that $f$ induces a continuous epimorphism $G / Z(G) \rightarrow H / Z(H)$. Hence $H \in[Z]$.

(3) If $G=\prod_{\alpha} G_{\alpha}$ then $Z(G)=\prod_{\alpha} Z\left(G_{\alpha}\right)$. Hence, as a topological group, $G / Z(G) \cong \prod_{\alpha}\left\{G_{\alpha} / Z\left(G_{\alpha}\right)\right\}$. Thus $G \in[Z]$ whenever each $G_{\alpha} \in[Z]$. The converse follows from (2).

(4) Denote the kernel of $f$ by $D$. Clearly, $f(Z(G)) \subseteq Z(H)$. In order to show the reverse inclusion assume $x \in G$ and $f(x) \in Z(H)$. Consider the continuous map $y \rightarrow x y x^{-1} y^{-1}$ of $G$ into $D$. Since $G$ is connected and $D$ is discrete, this map is constant; its value is clearly 1 . Hence $Z(H)=f(Z(G))$. Because $G$ is connected and $D$ is discrete and normal, we have $D \subseteq Z(G)$. Thus

$$
G / Z(G) \cong(G / D) /(Z(G) / D)=H / Z(H) .
$$

THEOREM 2.2. Let $G$ and $G^{*}$ be locally compact connected groups which are locally isomorphic. If $G \in[S I N]$ then $G^{*} \in[S I N]$.

Proof. Let $f: U \rightarrow U^{*}$ be the local isomorphism. In order to prove the theorem it suffices to show that every neighborhood $U_{1}^{*}$ of 1 in $G^{*}$ which is contained in $U^{*}$ contains an invariant neighborhood of 1 . Let $U_{1}=f^{-1}\left(U_{1}^{*}\right)$ and let $U_{2}$ be an invariant neighborhood of 1 contained in $U_{1}$. Then, since $f$ is a local isomorphism, one sees that $f\left(U_{2}\right)$ is a neighborhood of 1 in $G^{*}$ which is contained in $U_{1}^{*}$ and is normalized by $U_{1}^{*}$. Since $G^{*}$ is connected, the result follows.

$\left({ }^{5}\right)$ A further condition that ensures that $H \in[Z]$ is that $H$ be connected. This will be established in $\$ 4$ (Corollary 1 , Theorem 4.3). 
3. Extension theorems and applications. Let $G$ be a locally compact group, $\rho$ a continuous finite-dimensional, real representation of $G$ on $V$, and $N$ a closed normal subgroup of $G$. We denote by $V^{N}$ the $N$-fixed part of $V$.

Definition. (1) A continuous function $\psi: G \rightarrow V$ is called a crossed homomorphism if $\psi\left(g_{1} g_{2}\right)=\rho\left(g_{1}\right)\left(\psi\left(g_{2}\right)\right)+\psi\left(g_{1}\right)$, for all $g_{1}, g_{2}$ in $G$. (2) A continuous function $\phi: N \rightarrow V$ is called $G$-invariant if $\rho(g)(\phi(x))=\phi\left(g x g^{-1}\right)$, for all $x$ in $N$, $g$ in $G$.

Lemma 1. Let $G, N, \rho, V$ be as above. If $G / N$ is compact and $\phi$ is a $G$-invariant homomorphism on $N$ taking values in $V^{N}$ then $\phi$ extends to a crossed homomorphism $\psi: G \rightarrow V$.

For a proof, see $[1$, p. 22-01] or [5, p. 37, Proposition 2.2].

LEMMA 2. Let $G$ be a locally compact abelian group and $H$ a closed subgroup of $G$. If $\phi: H \rightarrow V$ is a continuous homomorphism, where $V$ is as above, then $\phi$ extends to a continuous homomorphism $\psi: G \rightarrow V$.

This fact was first proven by J. Dixmier in Bull. Sci. Math. 81 (1957); see also [12].

TheOREM 3.1. Let $G, N, \rho, V$ be as above and suppose $G \in[Z]$. If $\phi$ is a $G$-invariant homomorphism on $N$ mapping into $V^{N}$ and either $N$ or $Z$ is open in $G$ then $\phi$ extends to a crossed homomorphism $\psi$ on $G$.

Proof. Let $\phi_{1}=\left.\phi\right|_{N \cap Z}$. Clearly, $\phi_{1}$ is a $G$-invariant homomorphism. Since $N \cap Z$ is central, $\phi_{1}(N \cap Z) \subseteq V^{G}$. Now $V^{G}$ is a subspace of $V$. Hence, by Lemma 2 , $\phi_{1}$ extends to a continuous homomorphism $\phi_{2}: Z \rightarrow V^{G}$.

On the direct product $N \times Z$ we define a continuous homomorphism $\phi_{3}$ : $N \times Z \rightarrow V$ by $\phi_{3}(x, \hat{z})=\phi(x)+\phi_{2}(z)$. Since $Z$ is central there is a canonical epimorphism of $N \times Z$ onto $N Z$. Its kernel consists of the elements $\left(y, y^{-1}\right)$ with $y \in N \cap Z$. Obviously, these elements lie in the kernel of $\phi_{3}$. Hence $\phi_{3}$ induces a homomorphism $\phi_{4}: N Z \rightarrow V$ satisfying

$$
\phi_{4}(x z)=\phi(x)+\phi_{2}(z) .
$$

Now $N Z$ is a normal subgroup of $G$ containing $N$ and $Z$. Moreover, $\phi_{4}$ extends $\phi$ and $\phi_{2}$. Hence, if either $N$ or $Z$ is open in $G, \phi_{4}$ is continuous, because a homomorphism extending a continuous homomorphism on an open subgroup is itself continuous. It is easy to see that $\phi_{4}$ takes values in $V^{N Z}$ and that $\phi_{4}$ is $G$-invariant.

Clearly, $N Z$ is an open and hence closed subgroup of $G$. Since $G \in[Z], G / N Z$ is compact. By Lemma $1, \phi_{4}$ extends to a crossed homomorphism $\psi$ on $G$. Evidently, $\psi$ extends $\phi$.

THEOREM 3.2. (1) Let $G, N, \rho, V$ be as above and suppose $G \in[Z]$. If $N$ is central and $\phi: N \rightarrow V$ is a $G$-invariant homomorphism then $\phi$ extends to a crossed homomorphism $\psi$ on $G$. 
(2) Let $G, N, V$ be as above and suppose $G \in[Z]$. If $N$ is abelian and $\phi: N \rightarrow V$ is a continuous homomorphism satisfying $\phi\left(g x g^{-1}\right)=\phi(x)$, for all $x$ in $N, g$ in $G$, then $\phi$ extends to a continuous homomorphism $\psi$ on $G$.

Proof. (1) Since $N$ is central and $\phi$ is $G$-invariant, we have $\phi(N) \subseteq V^{G}$. By Lemma 2, $\phi$ extends to a continuous homomorphism $\phi_{1}: Z \rightarrow V^{G} \subseteq V$. Then $\phi_{1}$ is actually a $G$-invariant homomorphism. The result then follows from Lemma 1 .

(2) Since $Z$ is central and $N$ is normal, abelian, the closure $(N Z)^{-}$of $N Z$ in $G$ is a locally compact abelian group, normal in $G$. By Lemma $2, \phi$ extends to a continuous homomorphism $\phi_{1}:(N Z)^{-} \rightarrow V$. We show that $\phi_{1}\left(g t g^{-1}\right)=\phi_{1}(t)$, for all $t$ in $(N Z)^{-}$and $g$ in $G$. Clearly, it is sufficient to show this for $t$ in $N Z$. Thus let $t=x z$. Then, since $\phi_{1}$ is a homomorphism, and $Z$ is central, we have

$$
\phi_{1}\left(g x z g^{-1}\right)=\phi_{1}\left(\left(g x g^{-1}\right) z\right)=\phi_{1}\left(g x g^{-1}\right)+\phi_{1}(z) \text {. }
$$

Since $N$ is normal and $\phi_{1}$ extends $\phi$, this equals $\phi\left(g x g^{-1}\right)+\phi_{1}(z)$, which, by assumption, is $\phi(x)+\phi_{1}(z)$. On the other hand, $\phi_{1}(x z)=\phi(x)+\phi_{1}(z)$.

Note that $\phi_{1}$ is a $G$-invariant crossed homomorphism if we regard $V$ as a representation space for $G$ under the trivial representation. Since $G /(N Z)^{-}$is compact, Lemma 1 applies. This completes the proof.

Corollary 1. If $N$ is central and $\phi: N \rightarrow V$ is a continuous homomorphism then $\phi$ extends to a continuous homomorphism $\psi$ on $G$.

REMARK. In contrast to the abelian case, in general it is not possible to prove extension theorems analogous to Corollary 1 if the range of $\phi$ is not a vector group. To see this, consider the compact, simple group $\mathrm{SO}_{3}(R)$. Its universal covering group, $S U_{2}(C)$, has center $Z_{2}$, the integers modulo 2 . Let $\phi$ be the (natural) monomorphism of $Z_{2}$ into $\boldsymbol{T}$, the circle group. If $\phi$ extends to $\psi: S U_{2}(\boldsymbol{C}) \rightarrow \boldsymbol{T}$ then, by simple connectivity of $S U_{2}(C), \psi$ lifts to a continuous homomorphism $\chi$ : $S U_{2}(\boldsymbol{C}) \rightarrow \boldsymbol{R}$ such that exp $\circ \chi=\psi$. Since $S U_{2}(\boldsymbol{C})$ is compact and $\boldsymbol{R}$ has no nontrivial compact subgroups, $\chi$, and hence also $\psi$, is trivial. This is a contradiction.

COROLlaRY 2. If $V$ is a central vector subgroup of $G$, then $V$ is a direct factor.

Proof. Apply Corollary 1 to the identity map $V \rightarrow V$. The resulting extension $\psi: G \rightarrow V$ exhibits $V$ as a direct factor of $G$, because $V$ is central.

The remainder of this section deals with applications of the extension theorems to analytic groups.

Let $G$ be an analytic group, $\tilde{G}$ its universal covering group, and $D$ the kernel of the covering epimorphism. $D$ coincides with the fundamental group $\Pi_{1}(G)$. As is well known, since $G$ is an analytic group, $\Pi_{1}(G)$ is a finitely generated abelian group [5, p. 180, Theorem 3.1]. We denote its rank by $m$.

LEMMA 3. Suppose $G \in[Z]$. If $m \geqq 1$, then, as a manifold, $\tilde{G}=L \times \boldsymbol{R}^{m}$, where $L$ is a closed simply connected normal subgroup of $\tilde{G}$ containing $\tilde{G}^{\prime}$, the commutator subgroup of $\tilde{G}$. 
Proof. Theorem 2.1 guarantees that $\tilde{G} \in[Z]$, since $G \in[Z]$. By sending the finite part of $D$ to (0) and identifying the torsion free part of $D$ with its natural image in $\boldsymbol{R}^{m}$ we obtain a continuous homomorphism $\psi: D \rightarrow \boldsymbol{R}^{m}$, which, by Corollary 1, Theorem 3.2, extends to a continuous homomorphism $\psi: \widetilde{G} \rightarrow \boldsymbol{R}^{m}$. Since $\psi(\tilde{G})$ contains the lattice points, $\psi(\widetilde{G})=\boldsymbol{R}^{m}$.

Denote $\operatorname{Ker} \psi$ by $L$. Then $\widetilde{G} / L$ is isomorphic, as an analytic group, to $\boldsymbol{R}^{m}$. Now $L$ is a closed normal subgroup of $\tilde{G}$ containing $\tilde{G}^{\prime}$. Since $\tilde{G} / L$ is simply connected, $L$ must be connected. Hence, by a well-known theorem due to Mal'cev [10], $\widetilde{G}$ is isomorphic, as an analytic manifold, with $L \times(\tilde{G} / L)=L \times \boldsymbol{R}^{m}$.

THEOREM 3.3. If $G$ is an analytic group in $[Z]$ then

$$
\operatorname{rank} \prod_{1}(G) \leqq \operatorname{dim} G-\operatorname{dim} G^{\prime} .
$$

Proof. As above, denote rank $\prod_{1}(G)$ by $m$. If $m=0$ the inequality holds. If $m \geqq 1$ it follows from Lemma 3 that $m \leqq \operatorname{dim} \tilde{G}-\operatorname{dim} \tilde{G^{\prime}}$. Clearly, $\operatorname{dim} \tilde{G}=\operatorname{dim} G$ and $\operatorname{dim} \widetilde{G}^{\prime}=\operatorname{dim} G^{\prime}$.

ReMARK. In the case where $G$ is compact one knows that $m=\operatorname{dim} G-\operatorname{dim} G^{\prime}$.

CoRollary 2. (1) Let $G$ be any analytic group. Then rank $\prod_{1}(G)=\operatorname{dim} G$ if and only if $G$ is a torus.

(2) Let $G$ be an analytic group in $[Z]$. If $\operatorname{dim} G-\operatorname{rank} \prod_{1}(G) \leqq 2$ then $G$ is solvable.

Proof. (1) Let $Q$ be a maximal compact subgroup of $G$. Then rank $\Pi_{1}(G)$ $=\operatorname{rank} \prod_{1}(Q)$. Hence, by Theorem 3.J, rank $\prod_{1}(G) \leqq \operatorname{dim} Q-\operatorname{dim} Q^{\prime}$. Thus if rank $\prod_{1}(G)=\operatorname{dim} G$, then $G=Q$, so that $G$ is compact. Hence it follows from the above inequality that $\operatorname{dim} G^{\prime}=0$, i.e., that $G^{\prime}$ is discrete. Since it is connected, it must be trivial. Hence $G$ is a compact, abelian analytic group, i.e., a torus. The converse is clear.

(2) If $\operatorname{dim} G-\operatorname{rank} \prod_{1}(G) \leqq 2$ then $\operatorname{dim} G^{\prime} \leqq 2$. Thus $G^{\prime}$, and hence $G$, is solvable.

4. Basic structure theorems. In $\S \$ 4$ and 5 the following notational conventions will be used: $G_{1}$ denotes the identity component of $G, G^{\prime}$ the commutator subgroup, $\boldsymbol{T}^{n}$ the $n$-dimensional torus, $V$ and $W$ vector groups, $K$ a compact group; $\cong$ denotes an isomorphism of topological groups and - denotes topological closure.

In what follows $G$ will be locally compact. Denote by $\mathfrak{A}($ or $\mathfrak{A}(G)$ ) the group of all topological group automorphisms of $G$, by $M(C, U)$, where $C$ is a compact subset of $G$ and $U$ a neighborhood of 1 , the set of all $\alpha$ in $\mathfrak{A}$ such that

$$
\alpha(c) c^{-1} \in U \text { and } \alpha^{-1}(c) c^{-1} \in U,
$$

for every $c$ in $C$. As is known the family of all such $M(C, U)$ 's constitutes a fundamental system of neighborhoods of 1 of the group $\mathfrak{A}$ thus making $\mathfrak{A}$ a (not 
necessarily locally compact) topological group. We shall also have occasion to consider $\mathfrak{A}$ as a topological space in the compact-open topology; in this case we denote it by $\mathfrak{A}^{*}$. It is easy to see that the inclusion map $\mathfrak{A} \rightarrow \mathfrak{A}^{*}$ is continuous. As is known, if $G$ is compact, then $\mathfrak{U}=\mathfrak{U}^{*}$. Since the evaluation map $\mathfrak{H}^{*} \times G \rightarrow G$ is well known to be continuous, this implies that the evaluation $\mathfrak{A} \times G \rightarrow G$ is also continuous.

Proposition 4.1. The natural map $i: G \rightarrow \Im$ is a continuous homomorphism.

Proof. It suffices to check continuity at 1 . If $i_{x} \in M(C, U)$, then, for all $c$ in $C$,

$$
x c x^{-1} c^{-1} \in U \text { and } x^{-1} c x c^{-1} \in U .
$$

Choose a symmetric neighborhood $U^{\prime}$ of 1 such that $U^{\prime} U^{\prime} \subseteq U$. For every $c$ in $C$, choose a symmetric neighborhood $U_{c}$ of 1 such that $c U_{c}^{3} c^{-1} \subseteq U^{\prime}$. Since $C$ is compact, there exists a finite set $c_{1}, \ldots, c_{n}$ of elements of $C$ such that $\left\{c_{i} U_{c_{i}}\right\}$ covers $C$. Let $x$ belong to the neighborhood $U^{\prime} \cap\left(\bigcap_{i=1}^{n} U_{c_{i}}\right)$ of 1 in $G$, and let $c \in C$ (i.e., $c=c_{i} u_{i}$, where $u_{i} \in U_{c_{i}}$ ). Then

$$
x c x^{-1} c^{-1}=x c_{i}\left(u_{i} x^{-1} u_{i}^{-1}\right) c_{i}^{-1} \in U^{\prime} c_{i} U_{c_{i}}^{3} c_{i}^{-1} \subseteq U^{\prime} U^{\prime} \subseteq U .
$$

Similarly, $x^{-1} c x c^{-1} \in U$, and the proposition is proven.

For any family $\mathfrak{F}$ of continuous maps of $G$ into $G$, we denote by $\mathfrak{F}(x)$, where $x \in G$, the orbit of $x$ under $\mathfrak{F}$. The space of all continuous maps of $G$ into $G$, in the compact-open topology, will be denoted by $\mathfrak{M}$, the subspace consisting of endomorphisms by $\mathbb{E}$.

In the proof of the next theorem the following lemma will be needed.

LeMma 1. Assume that $G$ has small $\mathfrak{F}$-invariant neighborhoods of the identity, where $\mathfrak{F}$ is a subset of $\mathfrak{F}$. Let $\left\{f_{v}\right\}$ be a net in $\mathfrak{F}$ converging to $f$ and $\left\{g_{v}\right\}$ a net in $\mathfrak{M}$ converging to $g$, the convergence being taken with respect to the compact-open topology. Then $\left\{f_{v} \circ g_{v}\right\}$ converges to $f \circ g$.

Proof. Let $U$ be any neighborhood of 1 in $G$, and $C$ be any compact subset of $G$. Choose an $\mathfrak{F}$-invariant symmetric neighborhood $U^{\prime}$ of 1 satisfying the condition $U^{\prime} U^{\prime} \subseteq U$. For $c$ in $C$, we have

$$
\left(f_{v} \circ g_{v}\right)(c)((f \circ g)(c))^{-1}=\left(f_{v} \circ g_{v}\right)(c)\left(\left(f_{v} \circ g\right)(c)\right)^{-1}\left(f_{v} \circ g\right)(c)((f \circ g)(c))^{-1} \text {. }
$$

Since $G$ is locally compact, $g \in \mathfrak{M}$; therefore, $g(C)$ is compact. Hence there exists an index $\nu_{1}(C, U)$ after which $\left(f_{v} \circ g\right)(c)((f \circ g)(c))^{-1} \in U^{\prime}$. Now $\left(f_{v} \circ g_{v}\right)(c)$ $\left(\left(f_{v} \circ g\right)(c)\right)^{-1}=f_{v}\left(g_{v}(c)(g(c))^{-1}\right)$, so that there exists $\nu_{2}(C, U)$, after which this expression belongs to $f_{v}\left(U^{\prime}\right)$. However, since $U^{\prime}$ is $\mathfrak{F}$-invariant, this is contained in $U^{\prime}$. The result follows.

We are now in a position to state and prove Theorem 4.1 , which will be utilized in the proofs of some of our main theorems. 
TheOrem 4.1 (The Ascoli Theorem for Automorphism groups). Let $G$ be a locally compact group. A subgroup $\mathfrak{B}$ of $\mathfrak{A}(G)$ has compact closure if and only if the following holds:

(1) $(\mathfrak{B}(x))^{-}$is compact, for every $x$ in $G$.

(2) $G$ has small $\mathfrak{B}$-invariant neighborhoods of the identity.

Proof. Assume that $\overline{\mathfrak{B}}$ is compact. Then, in view of Proposition 4.1, $\tilde{\mathfrak{B}}$, the closure of $\mathfrak{B}$ in $\mathfrak{M}$, is compact. The Ascoli Theorem [9, p. 233] therefore implies that (1) and (2) hold.

The converse follows from the following lemma.

LEMma 2. Let $\mathfrak{B}$ be a subgroup of $\mathfrak{A}$. Assume that

(1) $\mathfrak{B}(x)$ is compact, for every $x$ in $G$.

(2) $G$ has small $\mathfrak{B}$-invariant neighborhoods of the identity.

Then $\tilde{\mathfrak{B}}$, the closure of $\mathfrak{B}$ in $\mathfrak{M}$, is a subgroup of $\mathfrak{A}$ and coincides with $\overline{\mathfrak{B}}$. Moreover, the two topologies agree on this set.

Proof. Let $\left\{\beta_{v}\right\}$ be a net in $\mathfrak{B}$ converging to an element $f$ of $\mathfrak{M}$, uniformly on compact subsets of $G$. By comparison with the point-open topology one sees easily that $f \in \mathfrak{F}$. Moreover, by comparison with the same topology, one sees that (1) and (2) hold if $\mathfrak{B}$ is replaced by $\tilde{\mathfrak{B}}$. Since $\tilde{\mathfrak{B}} \subseteq \mathfrak{F},(2)$, applied to $\tilde{\mathfrak{B}}$, is equivalent to equicontinuity of $\tilde{\mathfrak{B}}$ at every point of $G$. Hence, by the Ascoli Theorem $[9, \mathrm{p}$. 233], $\tilde{\mathfrak{B}}$ is compact. Since $\left\{\beta_{v}^{-1}\right\}$ is a net in $\tilde{\mathfrak{B}}$, this implies that there is a convergent subnet (which we denote by $\left\{\beta_{\mu}^{-1}\right\}$ ) converging to an element $g$ of $\tilde{\mathfrak{B}}$. Then, since $\beta_{\mu}$ converges to $f$, Lemma 1 shows that $f \circ g$ and $g \circ f$ equal the identity map of $G$ into $G$. Thus $\tilde{\mathfrak{B}} \subseteq \mathfrak{A}$.

The above proof also shows that $\tilde{\mathfrak{B}}=\tilde{\mathfrak{B}}^{-1}$. Now let $\gamma, \delta \in \tilde{\mathfrak{B}}$. There are nets $\left\{\beta_{v}\right\}$ and $\left\{\beta_{\mu}\right\}$ in $\mathfrak{B}$ converging to $\gamma$ and $\delta$, respectively, uniformly on compact sets. Choose a fixed index $\nu$. We show that the net $\left\{\beta_{\nu} \circ \beta_{\mu}\right\}$ converges (in $\mu$ ) to $\beta_{v} \circ \delta$. Let $C$ be a compact set and $U$ be a neighborhood of 1 in $G$; let $U^{\prime}$ be a $\mathfrak{B}$-invariant neighborhood of 1 contained in $U$. If $c \in C$ then we have

$$
\left(\beta_{v} \circ \beta_{\mu}\right)(c)\left(\left(\beta_{v} \circ \delta\right)(c)\right)^{-1}=\beta_{v}\left(\beta_{u}(c)(\delta(c))^{-1}\right) .
$$

There is a $\mu(C, U)$ after which $\beta_{\mu}(c)(\delta(c))^{-1} \in U^{\prime}$, for all $c$ in $C$. Thus the above expression is in $U$. On the other hand, $\left\{\beta_{\nu} \circ \delta\right\}$ converges (in $\nu$ ) to $\gamma \circ \delta$. For if $C$ and $U$ are as above, since $\gamma(C)$ is compact, there is a $\nu(C, U)$ after which

$$
\left(\beta_{v} \circ \delta\right)(c)((\gamma \circ \delta)(c))^{-1} \in U,
$$

for all $c$ in $C$. Hence, since the iterated $\operatorname{limit}_{\nu} \lim _{\nu} \lim _{\mu} \beta_{\nu} \circ \beta_{\mu}$ exists, there is a net $\left\{\beta_{\nu} \circ \beta_{\mu(v)}\right\}$ converging to $\gamma \circ \delta[9$, p. 69]. Since $\mathfrak{B}$ is a group, $\gamma \circ \delta \in \tilde{\mathfrak{B}}$.

Obviously, $\overline{\mathfrak{B}} \subseteq \tilde{\mathfrak{B}}$. Let $\left\{\gamma_{v}\right\}$ be a net in $\tilde{\mathfrak{B}}$ converging to $\gamma$, uniformly on compact sets. We claim that then $\left\{\gamma_{v}^{-1}\right\}$ converges to $\gamma^{-1}$. For let $C$ be a compact set and $U$ be a neighborhood of 1 in $G$. Since $\gamma^{-1}(C)$ is compact, and since $U$ contains a $\tilde{\mathfrak{B}}$-invariant neighborhood $U^{\prime}$ of 1 we have

$$
\gamma_{v}\left(\gamma^{-1}(c)\right)\left(\gamma\left(\gamma^{-1}(c)\right)\right)^{-1} \in U^{\prime},
$$


for every $c$ in $C$ and all $\nu$ after a fixed index $\nu(C, U)$. Hence

$$
\gamma^{-1}(c)\left(\gamma_{v}^{-1}(c)\right)^{-1} \in U^{\prime}
$$

so that

$$
\gamma_{v}^{-1}(c)\left(\gamma^{-1}(c)\right)^{-1} \in U
$$

for all $c$ in $C$ and $\nu$ after $\nu(C, U)$.

This proves the assertion. In particular, this shows that $\tilde{\mathfrak{B}} \subseteq \overline{\mathfrak{B}}$, so that $\overline{\mathfrak{B}}=\tilde{\mathfrak{B}}$. Finally, the above shows that the two topologies coincide on this set. This completes the proof.

Part (1) of the next theorem is a direct consequence of the preceding results in combination with the Open Mapping Theorem [17]; part (2) follows from Theorem 4.1.

THEOREM 4.2( $\left.{ }^{6}\right)$. (1) If $G \in[Z]$ then

(a) $G \in[S I N]$;

(b) $\Im(x)$ is compact, for every $x$ in $G\left({ }^{7}\right)$.

Conversely, assume that (a) and (b) hold. If $\mathfrak{3}$ is closed in $\mathfrak{A}$ and if $G$-or even $G / Z$-is $\sigma$-compact then $G \in[Z]$.

(2) If $G$ is a locally compact group then $\bar{\Im}$ is compact if and only if

(a) $G \in[S I N]$;

(b) $(\Im(x))^{-}$is compact, for every $x$ in $G\left(^{8}\right)$.

Now we turn to the structure theorems.

THEOREM 4.3. If $G$ is a locally compact, connected group then the following conditions are equivalent:

(1) $G \in[Z]$.

(2) $G \in[S I N]$.

(3) $G=V \times K$ (direct product).

Proof. By Theorem 4.2, (1) implies (2). Now suppose $G \in[S I N]$. If, in addition, $G$ is an analytic group, (3) follows readily from a theorem of Mostow [13]. However, the following argument yields a direct proof:

Since $G \in[S I N]$ it follows from the commutativity relation $i_{x} \circ \exp =\exp \circ i_{x}$ (where $i_{x}$ is the differential of the inner automorphism $i_{x}$ ) that $G^{\cdot}$, the Lie algebra of $G$, regarded as an abelian topological group, has small $\alpha(G)$-invariant neighborhoods of 1 , where $\alpha$ denotes the adjoint representation of $G$ on $G$. Moreover, since the elements of $\alpha(G)$ are linear maps, the orbits of points of $G$. (with respect to $\alpha(G)$ )

( $\left.{ }^{6}\right)$ Part (2) of this theorem provides the justification of an assertion made in $[4$, p. 588] where R. Godement states that the class of discrete $[F I A]^{-}$-groups coincides with the class of discrete $F C$-groups. As Theorems 4.1 and 4.2 show, this is true, although not completely obvious.

$\left({ }^{7}\right)$ Hence $G$ is a topological $F C$-group.

$\left.{ }^{(}\right)$Hence $G$ is a topological $(F C)^{-}$-group. 
have compact closure in $G$. Hence, by Theorem $4.1, \alpha(G)$ has compact closure, $\mathfrak{B}$ say, in $\mathfrak{A}\left(G^{\cdot}\right)$, the general linear group. Denote the normalized Haar integral on $\mathfrak{B}$ by $\hat{\rfloor}_{\mathfrak{B}} d \beta$. If $(\xi, \eta)$ denotes an inner product on $G \cdot$, then

$$
\langle\xi, \eta\rangle=\int_{\mathfrak{B}}(\beta(\xi), \beta(\eta)) d \beta
$$

defines an inner product on $G$, which is $G$-invariant. Hence [5, Theorem 1.1, p. 142] $G^{\cdot}$ is the Lie algebra of a compact analytic group, so that [5, Theorem 1.3, p. 144] $G^{\cdot}=\left[G^{*}, G^{\cdot}\right] \oplus Z\left(G^{*}\right)$, where $Z\left(G^{*}\right)$ denotes the center of $G^{\cdot}$ and $\left[G^{*}, G^{*}\right]$ is the Lie algebra of a compact, semisimple analytic group. It follows that $G=G^{\prime} Z(G)_{1}$, where $G^{\prime}$ is the commutator subgroup of $G$. Since $G^{\prime}$ is locally isomorphic to a compact analytic group, their common universal covering group is compact, by Weyl's Theorem. Thus $G^{\prime}$ is itself compact. On the other hand, $Z(G)_{1}=T^{n} \times V$. Thus $G=V K$, where $K=G^{\prime} T^{n}$ is a compact group. Since $K \cap V=(1)$ and $V$ is central, (3) follows $\left({ }^{9}\right)$.

Now let $G$ be any locally compact, connected group satisfying (2). For each neighborhood $U$ of 1 in $G$, choose a compact normal subgroup $H_{U}$ of $G, H_{U} \subseteq U$, such that $G / H_{U}$ is an analytic group [11, p. 175]. It is easily seen that $G / H_{U}$ obeys (2) and hence, by the above, is the direct product of a vector group and a compact group. Hence $G / H_{U}$ is representable in a compact group $C_{U}$, by a continuous monomorphism $f_{U}$. Let $C=\prod_{U} C_{U}$ and define $f: G \rightarrow C$ by

$$
f(x)_{U}=\left(f_{U} \circ \pi_{U}\right)(x)
$$

where $\pi_{U}: G \rightarrow G / H_{U}$ is the canonical epimorphism. Then $f$ is a continuous homomorphism and, evidently, $\operatorname{Ker} f=\bigcap_{U} H_{U}$. Thus $\operatorname{Ker} f=(1)$, since $H_{U} \subseteq U$, so that $G$ is representable in a compact group. By a theorem of FreudenthalWeil [5, Theorem 2.1, p. 145] this proves (3). (3) implies (1), by Theorem 2.1.

REMARK. The above result on the structure of connected groups in $[Z]$ is also an immediate consequence of facts on representations of groups in $[Z]$ in combination with the theorem of Freudenthal-Weil. In the case of connected, second countable $G$ a proof was given by Freudenthal.

Corollary 1. If $G \in[Z]$ and $H$ is a closed, connected subgroup of $G$ then $H \in[Z]$.

Proof. $G$ has small invariant neighborhoods of 1 , by Theorem 4.2. Hence $H$ has the same property. Since $H$ is connected, the result follows.

COROLlARY 2. Let $G$ and $G^{*}$ be locally compact connected groups which are locally isomorphic. If $G \in[Z]$ then $G^{*} \in[Z]$.

This follows directly from Theorems 4.3 and 2.2.

(9) We thank C. Moore for a helpful suggestion in this proof. 
COROllaRY 3. If $G$ is an analytic group in [Z] (in particular, if $G$ is a compact analytic group) then any covering group of $G$ is the direct product of a vector group and a compact group $\left({ }^{10}\right)$.

Proof. This follows for instance immediately from Theorem 4.3, and Theorem 2.1.

Theorem 4.4 (Structure Theorem). Let $G \in[Z]$. Then $G=W \times H$ (direct product), where $W$ is a vector group and $H$ contains a compact, open, normal subgroup $K$.

The proof of Theorem 4.4 will be given in several stages $\left({ }^{11}\right)$.

LEMMA 1. Let $A$ be a locally compact, connected abelian group and $\mathfrak{F}$ a finite group of topological group automorphisms of $A$. Then $A=V \times L$, where $V$ is $\mathfrak{F}$-stable and $L$ is compact $\left({ }^{12}\right)$.

Proof. As is well known, $A=W \times L$, where $W$ is a vector group and $L$ is compact. Let $n$ denote the order of $\mathfrak{F}$. For $\sigma$ in $\mathfrak{F}$ and $w$ in $W$, write $\sigma(w)=\omega_{\sigma}(w)+\lambda_{\sigma}(w)$, where $\omega_{\sigma}(w) \in W, \lambda_{\sigma}(w) \in L$. Evidently, for $\sigma, \tau$ in $\mathfrak{F}, \omega_{\sigma} \circ \omega_{\tau}=\omega_{\sigma \tau}$. Define the $\operatorname{map} \alpha: A \rightarrow A$ by

$$
\alpha(w+l)=\sum_{\sigma} \sigma\left(\omega_{\sigma^{-1}}(w / n)\right)+l .
$$

One sees easily that, for $\tau$ in $\mathfrak{F}$, the formula $\tau(\alpha(w))=\alpha\left(\omega_{\tau}(w)\right)$ holds. Thus $\alpha(W)$ is $\mathfrak{F}$-stable. By the above,

$$
\alpha(w+l)=w+\left[\sum_{\sigma} \lambda_{\sigma}\left(\omega_{\sigma^{-1}}(w / n)\right)+l\right] .
$$

Evidently, the map $\beta: A \rightarrow A$, defined by

$$
\beta(w+l)=w+\left[\sum_{\sigma} \lambda_{\sigma}\left(\omega_{\sigma}-1(-w / n)\right)+l\right]
$$

is the inverse of $\alpha$, so that $\alpha$ is bijective. One checks directly from the definition of $\alpha$ that $\alpha$ is a continuous homomorphism. Since $\beta=\alpha^{-1}$ is also continuous, $\alpha$ is a topological group automorphism. Thus $\alpha(W)$ is a vector group, $V$ say. Since $A=W \times L$ and $L$ is fixed under $\alpha, A=V+L$. Since $L$ is compact and $V$ is a vector group, this implies $A=V \times L$.

LeMma 2. (1) Let $G$ be any topological group. Then $Z\left(G_{1}\right)_{1}$ is normal in $G$.

(2) If $G$ is a group in $[Z]$ and $G / G_{1}$ is compact then $G / Z\left(G_{1}\right)_{1}$ is compact.

$\left({ }^{10}\right)$ The same fact was proven in [1, p. 22-05].

( ${ }^{11}$ ) In the course of proving Theorem 4.4 (particularly, in Lemma 4) we received substantial help from Professor G. P. Hochschild, for which we express our gratitude.

$\left({ }^{12}\right)$ We are aware of the fact that a generalized form of Lemma 1 ( $\&$ compact) is to be found in the literature. However, for reasons which we will make clear in a subsequent publication, neither one of the two proofs that have been published can be considered as cogent. 
Proof. (1) is trivial. To prove (2), it obviously suffices to show that $G_{1} / Z\left(G_{1}\right)_{1}$ is compact. By Corollary 1 , Theorem 4.3, $G_{1} \in[Z]$. Hence $G_{1}=W \times K$, by Theorem 4.3. Thus $Z\left(G_{1}\right)_{1}=(W \times Z(K))_{1}=W \times Z(K)_{1}$, so that $G_{1} / Z\left(G_{1}\right)_{1} \cong K / Z(K)_{1}$. The result follows.

LEMMA 3. Let $G \in[Z]$ and suppose $G$ is a semidirect product $V \times{ }_{n} H$, where $V$ is a normal vector subgroup of $G$. (For notation and terminology concerning semidirect products see $[5$, pp. 39, 42].) Then $V$ is a direct factor of $G$.

Proof. Note that $\eta$ takes values in the group of linear automorphisms of $V$. A straightforward computation shows that $Z\left(V \times{ }_{\eta} H\right)$ is the set of all pairs $(v, h)$ where $v \in V^{H}$, the $H$-fixed part of $V$, and $h \in Z(H) \cap \operatorname{Ker} \eta$. Define a map

$$
f:\left(V \times{ }_{\eta} H\right) / Z\left(V \times{ }_{\eta} H\right) \rightarrow V / V^{H} \text { by } f\left\{(v, h) Z\left(V \times{ }_{\eta} H\right)\right\}=v+V^{H} .
$$

If $\left(v_{1}, h_{1}\right) \in Z\left(V \times{ }_{\eta} H\right)$ then

$$
f\left((v, h)\left(v_{1}, h_{1}\right)\right)=f\left(v+h \cdot v_{1}, h h_{1}\right)=\left(v+h \cdot v_{1}\right)+V^{H}=v+V^{H},
$$

which shows that $f$ is well defined. Clearly, $f$ is a continuous map. Thus $V / V^{H}$ is compact. Since $V^{H}$ is a subspace of $V$, it follows that $V=V^{H}$, so that $G=V \times H$.

LeMma 4. Let $G \in[Z]$ and suppose that $G / G_{1}$ is compact. Then $G=V \times K$.

Proof. Assume first that $G$ is a Lie group. Since $Z\left(G_{1}\right)_{1}$ is central in $G_{1}$, it is fixed under inner automorphisms of $G_{1}$. By Lemma 2, (1), $Z\left(G_{1}\right)_{1}$ is normal in $G$. Hence the finite group $G / G_{1}$ acts on $Z\left(G_{1}\right)_{1}$, and Lemma 1 applies. Thus $Z\left(G_{1}\right)_{1}=V \times T^{n}$, where $V$ is stable under $G / G_{1}$ and hence under $G$.

Now $(G / V) /\left(Z\left(G_{1}\right)_{1} / V\right) \cong G / Z\left(G_{1}\right)_{1}$. By Lemma $2,(2), G / Z\left(G_{1}\right)_{1}$ is compact. Since $Z\left(G_{1}\right)_{1} / V \cong T^{n}, G / V$ is compact. Hence it follows from [1, p. 22-04] or $[5$, p. 39] that $V$ is a semidirect factor of $G$. Thus, by Lemma 3, $V$ is a direct factor of $G$, so that $G=V \times K$, and $K \cong G / V$ is compact.

In the general case, since $G / G_{1}$ is compact, there is a compact normal subgroup $H$ of $G$ such that $G / H$ is a Lie group. (See $[11$, p. 175].) It is easy to see that $G / H$ has a finite number of components. Also $G / H \in[Z]$, by Theorem 2.1 . Hence, by the above, $G / H=W \times L$, where $L$ is compact. Let $K$ be the inverse image of $L$ in $G$ under the canonical epimorphism $G \rightarrow G / H$. $K$ is a closed normal subgroup of $G$, and is compact, because $H$ and $L$ are compact. Moreover,

$$
G / K \cong(G / H) /(K / H) \cong W
$$

so that $G / K$ is a vector group. Hence $K$ contains every compact subgroup of $G$. By a well-known theorem of Iwasawa [Ann. of Math. (2) 50 (1949), 507-558; p. 509], since $K$ is compact, $\mathfrak{A}(K) / \Im(K)$ is totally disconnected.

Now the map $G \rightarrow \mathfrak{A}(K)$, defined by $\left.x \rightarrow i_{x}\right|_{K}$, induces a continuous homomorphism $G / K \rightarrow \mathfrak{A}(K) / \Im(K)$, which is trivial since $G / K$ is connected. This means 
that, for $x$ in $G,\left.i_{x}\right|_{K} \in \Im(K)$. Thus $x k_{1} x^{-1}=k k_{1} k^{-1}$, for some $k$ and all $k_{1}$ in $K$. It follows that $k^{-1} x \in C(K)$, the centralizer of $K$ in $G$; therefore $G=K C(K)\left({ }^{13}\right)$.

By restriction, the continuous epimorphism $G \rightarrow W$ gives rise to a continuous homomorphism $f: C(K) \rightarrow W$. Since $K$ is compact, $f$ is a surjective, open, and closed map. Therefore, $f\left(C(K)_{1}\right)$ is a closed, connected subgroup of $W$, i.e., a vector subgroup, say $W^{*}$, and the resulting induced map $f^{*}: C(K) / C(K)_{1} \rightarrow W / W^{*}$ is a continuous, open epimorphism. Since $C(K) / C(K)_{1}$ is locally compact and totally disconnected, any neighborhood of 1 contains a compact, open subgroup. Let $S$ denote any such subgroup. Now $W / W^{*}$ is a vector group, so that $f^{*}(S)=(1)$, i.e., the kernel of $f^{*}$ is open. Since $f^{*}$ is open, we have $W / W^{*} \cong\left(C(K) / C(K)_{1}\right) / \operatorname{Ker} f^{*}$, which is discrete. Thus $W=W^{*}$, so that $G=C(K)_{1} K$.

Since $C(K)_{1}$ is a closed, connected subgroup of $G$, and $G \in[Z], C(K)_{1} \in[Z]$, by Corollary 1, Theorem 4.3. Thus $C(K)_{1}=V \times M$, where $M$ is compact (Theorem 4.3). But $M \subseteq K$. Hence $G=V K$. Also, $V \cap K=(1)$. Since $V \subseteq C(K), V$ and $K$ commute pointwise.

LEMMA 5. If $G$ is an arbitrary group in $[Z]$ then $G \supseteq L$, an open normal subgroup, and $L=V \times K$ (direct product).

Proof. Let $G_{2}=G / G_{1}$. Since $G_{2} \in[Z]$ (by Theorem 2.1), $G_{2}$ has small invariant neighborhoods of 1 (Theorem 4.2, (1)). Moreover, since $G_{2}$ is locally compact and totally disconnected, any neighborhood $U_{2}$ of 1 contains a compact, open subgroup $H_{2}$. There exists an invariant neighborhood $V_{2}$ of $1, V_{2} \subseteq H_{2}$. Hence $V_{2} \subseteq L_{2}$, where $L_{2}=\bigcap_{x_{2} \in G_{2}} x_{2} H_{2} x_{2}^{-1}$. Thus $L_{2}$ is a compact, open, normal subgroup of $G_{2}$. Let $L=\pi^{-1}\left(L_{2}\right)$, where $\pi: G \rightarrow G_{2}$ is the canonical epimorphism. $L$ is an open, normal subgroup of $G$, so that $L_{1}=G_{1}$. Hence $L / L_{1}=\pi^{-1}\left(L_{2}\right) / G_{1}=L_{2}$, and $L / L_{1}$ is compact. By Theorem 2.1, $L \in[Z]$, because $L$ is open in $G$. Then Lemma 4 applies to $L$, and the proof of Lemma 5 is complete.

LeMmA 6. If $G \in[Z]$ and $V$ is an open normal vector subgroup of $G$ then $V$ is central in $G$.

Proof. Obviously, $V Z / Z$ is a closed subgroup of $G / Z$, hence is compact. Since $V$ is open in $G$, we have $V Z / Z \cong V / V \cap Z$. Since $V$ is normal, $G$ acts on $V$ by the adjoint representation. This is a linear representation, and $V \cap Z=V^{G}$ is a subspace of $V$. Hence $V=V \cap Z$, so that $V \subseteq Z$.

Proof of Theorem 4.4. Let $L, V, K$ be the groups obtained in Lemma 5 . Since $L$ is normal in $G,\left.i_{x}\right|_{L} \in \mathfrak{A}(L)$, for every $x$ in $G$. Clearly, $Z(L)_{1}$ is a characteristic $\left({ }^{14}\right)$ subgroup of $L$, so that the restriction of $i_{x}$ to $Z(L)_{1}$ belongs to $\mathfrak{A}\left(Z(L)_{1}\right)$.

As usual, denote $Z(G)$ by $Z$. Now $Z L$ is an open, normal subgroup of $G$ containing $L$. Hence $G / Z L$ is finite. Moreover, $Z L$ centralizes $Z(L)_{1}$, so that $G / Z L$ acts as a

${ }^{(13)}$ Actually, this fact is proven directly in [2].

$\left({ }^{14}\right)$ The notion of characteristic subgroup is defined with reference to topological group automorphisms. 
(finite) group of automorphisms of the locally compact, connected, abelian group $Z(L)_{1}$. Since $L=V \times K, Z(L)_{1}=V \times Z(K)_{1}$, so that $Z(K)_{1}$ is the maximum compact subgroup of $Z(L)_{1}$. By Lemma $1, Z(L)_{1}=W \times Z(K)_{1}$, where $W$ and (clearly) $Z(K)_{1}$ are $G / Z L$-stable, and therefore $G$-stable.

Obviously, $\operatorname{dim} V=\operatorname{dim} W$. Since $K$ is compact and normal in $L$ and $W$ is closed and central, $W K$ is a closed normal subgroup of $L$.

Furthermore, $L / W K \cong(L / K) /((W K) / K)$, and since $K$ is compact, $W K / K$ $\cong W / W \cap K \cong W$. Thus $L / W K \cong V / W=(1)$, so that $L=W K$. Thus $L=W \times K$.

Since $W$ is central in $L$, the centralizer $L^{*}$ of $W$ in $G$ contains $Z L$, hence is of finite index in $G$. By Theorem 2.1, $L^{*} \in[Z]$. Hence $W$ is a direct factor of $L^{*}$, by Corollary 2, Theorem 3.2. Thus $L^{*}=W \times H$, where $H \cong L^{*} / W$, and $K \cong L / W$ is an open, normal subgroup of $L^{*} / W$. Hence $H$ contains a compact, open, normal subgroup, which we again denote by $K$.

We write $G=\bigcup_{i=1}^{N} x_{i} L^{*}$, where $N$ is the index of $L^{*}$ in $G$. Since $H$ is a direct factor of $L^{*}$ and $K$ is normal in $H, K$ is normal in $L^{*}$. It follows that $\bigcap_{i=1}^{N} x_{i} K x_{i}^{-1}$ $=\bigcap_{x \in G} x K x^{-1}$. Denote this compact, normal subgroup of $G$ by $K^{*}$. Now, for any $i, x_{i} K x_{i}^{-1}$ is a compact subgroup of $L^{*}$, hence is annihilated by the projection $L^{*} \rightarrow W$, since $W$ is a vector group. Consequently, $x_{i} K x_{i}^{-1} \subseteq H$, so that $K^{*}$ is an open subgroup of $H$.

Let $\pi$ be the canonical epimorphism $G \rightarrow G / K^{*}$. Since $W \times K^{*}$ is open in $L^{*}$, it is open in $G$. Then $\pi\left(W \times K^{*}\right)$ is an open vector subgroup, $W^{*}$ say, of $G / K^{*}$. It follows from Lemma 6 that $W^{*} \subseteq Z\left(G / K^{*}\right)$, since $G / K^{*} \in[Z]$. The last result means that $g w g^{-1} w^{-1} \in K^{*}$, for all $g$ in $G, w$ in $W$. On the other hand, since $W$ is normal in $G, g^{\prime} g^{-1} w^{-1} \in W$. Hence $g w g^{-1} w^{-1} \in W \cap K^{*}=(1)$, so that $W$ is central in $G$. Hence $G=L^{*}=W \times H$. Q.E.D.

Corollary 1. If $G \in[Z]$ then $\left(G^{\prime}\right)^{-}$is compact.

Proof. It follows from Theorem 4.4 that $G^{\prime}=H^{\prime}$, and since $H$ is closed in $G$, $\left(G^{\prime}\right)^{-}=\left(H^{\prime}\right)^{-}$. Hence we may assume that $G=H$, i.e., that $G$ contains a compact, open, normal subgroup $K$. Then $G / K$ is a discrete group whose center is of finite index. This implies that $(G / K)^{\prime}$ is finite $[14$, p. 186]. The inverse image in $G$ of $(G / K)^{\prime}$ is $G^{\prime} K$. It follows immediately that $\left(G^{\prime}\right)^{-}$is compact.

A straightforward application of Corollary 1 to the situation of Theorem 4.4 yields

Corollary 2. If $G \in[Z]$ then $G=W \times H$ (direct product) where $H$ contains a compact, open, normal subgroup $K$ and $H / K$ is abelian.

We now prove the following approximation theorem.

THEOREM 4.5. If $G \in[Z]$ then $G$ is a projective limit of Lie groups.

Proof. Consider the exact sequence

$$
\text { (1) } \longrightarrow G_{1} \longrightarrow G \stackrel{\pi}{\longrightarrow} G_{2} \longrightarrow \text { (1). }
$$


As in the proof of Lemma 5 we obtain an open normal subgroup $L$ of $G$ such that $L / L_{1}$ is compact.

Let $U$ be a neighborhood of 1 in $G$. Since $L$ is open in $G$, by taking $L \cap U$, if necessary, we may assume that $U$ is a neighborhood of 1 in both $G$ and $L$. Since $L / L_{1}$ is compact, there exists a compact, normal subgroup $L_{*}$ of $L$ such that $L_{*} \subseteq U$ and $L / L_{*}$ is a Lie group [11, p. 175]. Since $L$ is open, $L Z$ is an open subgroup of $G$, which is normal, because $L$ is normal and $Z$ is central. Hence $G=\bigcup_{i=1}^{N} x_{i} L Z$, for some finite number of points $x_{i}$ of $G$.

Denote the normal subgroup $\bigcap_{x \in G} x L_{*} x^{-1}$ of $G$ by $L_{* *}$. A direct calculation, using the above expression for $G$ as well as the fact that $L Z$ normalizes $L_{*}$, now shows that $L_{* *}=\bigcap_{i=1}^{N} x_{i} L_{*} x_{i}^{-1}$. Since $L_{* *}$ is a closed subgroup of $L_{*}$, it is compact and contained in $U$. Now define $L_{*}^{i}$ to be $x_{i} L_{*} x_{i}^{-1}$, for $i=1, \ldots, N$. Since $L$ is normal in $G, L_{*}^{i}$ is the transform of $L_{*}$ by an automorphism of $L$; thus $L_{*}^{i}$ is closed and normal in $L$.

Obviously, for each $i, i_{x_{i}}$ induces a topological group isomorphism of $L / L_{*}$ with $L / L_{*}^{i}$. Hence each $L / L_{*}^{i}$ and therefore $\prod_{i=1}^{N}\left(L / L_{*}^{i}\right)$ is a Lie group.

Now consider the map $f: L \rightarrow \prod_{i=1}^{N}\left(L / L_{*}^{i}\right)$ defined by $f(l)=\left(\pi^{1}(l), \ldots, \pi^{N}(l)\right)$, where $\pi^{i}: L \rightarrow L / L_{*}^{i}$ is the canonical epimorphism. Evidently, $f$ is a continuous homomorphism, with kernel $L_{* *}$, and induces a continuous monomorphism of $L / L_{* *}$ into $\prod_{i=1}^{N}\left(L / L_{*}^{i}\right)$. Since $L / L_{* *}$ is locally compact, this implies, by a wellknown theorem of Cartan, that it is a Lie group. Hence both $L / L_{* *}$ and $\left(G / L_{* *}\right) /\left(L / L_{* *}\right) \cong G / L$ are Lie groups, since the latter is a discrete group. It follows from [2, p. 90] that $G / L_{* *}$ is a Lie group. This completes the proof.

We now carry out the analysis announced in the Introduction. The results will be given in the form of a diagram.

EXAMPLE I $\left({ }^{15}\right)$. Let $G$ be the group of all $3 \times 3$ real matrices of the form

$$
\left[\begin{array}{lll}
1 & 0 & 0 \\
m & 1 & 0 \\
x & n & 1
\end{array}\right],
$$

where $m, n \in Z$. It is easily seen that $Z(G)=G_{1}=R$. From this it follows immediately that $G \notin[Z]$, but $G \in[S I N]$. Moreover, there is no semidirect product decomposition of the form $G=V \times{ }_{\eta} H$. Thus groups in [SIN] do not even satisfy this weaker structure theorem. This conclusion cannot be drawn from the examples referred to in the Introduction. Using Theorem 4.2 one sees easily that $G \notin[F I A]^{-}$.

EXAMPLE II (adapted from [4]). Let $G$ be the discretely topologized weak direct sum of countably many copies of a finite, simple group $K$. It is easy to see that $Z(G)=(1)$, that the conjugacy classes of $G$ are finite, and that $G^{\prime}=G$. By Theorem 4.2, $G \in[F I A]^{-}$.

EXAMPLE III. In [13, p. 344] G. D. Mostow gave an example of an analytic

(15) This example was pointed out to us by Professor G. P. Hochschild. 
group which established the distinction between $[I N]$ and $[S I N]$, thus correcting an error in [19, p. 129].

In the diagram below the symbol $[X] \rightarrow m(n)[Y]$ denotes proper inclusion of $[X]$ in $[Y]$, the reason for the inclusion being given by $m$, and the properness given by $n$. The appearance of the letter $c$ or $d$ indicates that the relations hold only in the case of connected or discrete groups, respectively, and $\stackrel{c}{=}$ denotes equality in the case of connected groups.

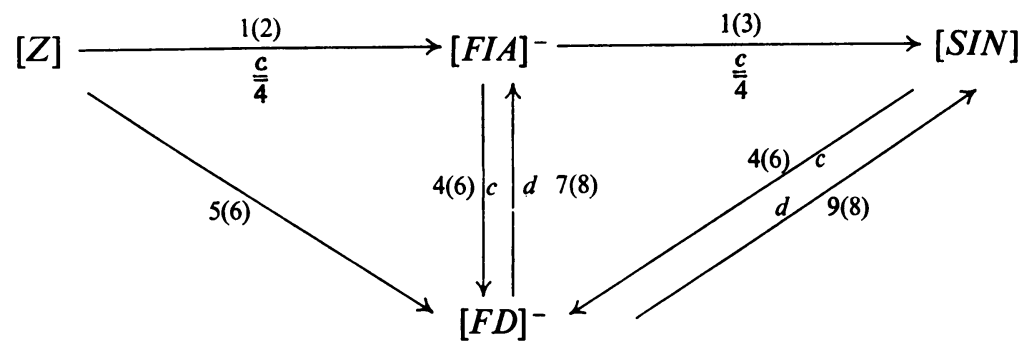

1. Theorem 4.2.

2. Example II.

3. Example I.

4. Theorems 4.2 and 4.3.

5. Theorem 4.4, Corollary 1.

6. Iwasawa's Theorem [7, p. 346], Example III and Theorem 4.2.

7. Neumann's Theorem [14, p. 186] and Theorem 4.2.

8. Example II.

9. Trivial.

5. Applications of the structure theorems. The notational conventions introduced in $\$ 4$ are still in force.

THEOREM 5.1. Let $G$ be a connected group in $[Z]$. Then the set of points of $G$ that lie on one-parameter subgroups is dense in $G$.

Proof. The result is obvious for vector groups and well known for compact connected groups [11]; hence, in view of Theorem 4.3, it holds for connected $[Z]$-groups.

Corollary. A connected $[Z]$-group $G$ is divisible $\left({ }^{16}\right)$.

Proof. Since vector groups are divisible, Theorem 4.3 allows one to reduce the proof to the case where $G$ is compact. If $f_{n}: G \rightarrow G$ denotes the map $x \rightarrow x^{n}$ for a fixed (but arbitrary) integer $n$, then, since $f_{n}(G)$ is clearly compact, it suffices (in view of Theorem 5.1) to show that $f_{n}(G)$ contains the set of points of $G$ that lie on one-parameter subgroups. This, in turn, follows easily from the divisibility of $\boldsymbol{R}$.

$\left({ }^{16}\right)$ We are indebted to the referee for suggesting this corollary. 
THEOREM 5.2. If $G \in[Z]$ and $V$ is a normal vector subgroup of $G$ then $V$ is a direct factor.

Proof. By Theorem 4.4, $G=W \times H$, where $H \supseteq K$. Let $\pi_{W}$ and $\pi_{H}$ be the corresponding projections. If $g \in G$ write $g=\pi_{W}(g) \pi_{H}(g)$. It is obvious that $V \cap H$ $\subseteq \operatorname{Ker}\left(\left.\pi_{W}\right|_{v}\right)$. On the other hand, $\operatorname{Ker}\left(\left.\pi_{W}\right|_{V}\right) \subseteq V \cap K=(1)$. For let $\pi_{W}(v)=1$. Then $v=\pi_{H}(v) \in \pi_{H}(V)$, which is in $H_{1}$, since $V$ is connected. But $H_{1}=K_{1}$, since $K$ is open in $H$. Thus $V \cap H=(1)$.

Now assume $g \in G, v \in V$. Then

$$
\pi_{W}\left(g v g^{-1} v^{-1}\right)=\pi_{W}(g) \pi_{W}(v) \pi_{W}(g)^{-1} \pi_{W}(v)^{-1}=1,
$$

because $W$ is abelian. Thus $g v g^{-1} v^{-1}$ belongs to $H$; on the other hand, it belongs to $V$, because $V$ is normal. This proves that $V$ is central. The result now follows from Corollary 2, Theorem 3.2.

The next theorem is a consequence of Corollary 1 of Theorem 4.4 and an extension theorem.

THEOREM 5.3. Suppose $G$ is a connected [Z]-group and $D$ a discrete normal subgroup. Let $(1) \rightarrow D \rightarrow G \rightarrow H \rightarrow(1)$ be the corresponding group extension. If $H^{\prime}$ is dense in $H$ then $G$ is compact.

Proof. Since $D$ is necessarily central, it is a discrete subgroup of the compactly generated abelian group $V \times Z(K)$, where $G=V \times K$ according to Theorem 4.3. Hence, as can be shown by abelian group theory, $D$ is compactly, i.e., finitely, generated. Thus $D$ is a finitely generated abelian group. If its rank is $n \geqq 1$, the construction used in the proof of Lemma 3 of $\S 3$, with $G$ in the place of $\tilde{G}$, yields a continuous homomorphism $\psi: G \rightarrow \boldsymbol{R}^{n}$. Since $\psi(G)$ is connected, it does not coincide with $\boldsymbol{Z}^{n}$, the set of lattice points of $\boldsymbol{R}^{n}$. However, $\psi(D)=\boldsymbol{Z}^{n}$, so that $\psi$ induces a nontrivial continuous homomorphism from $H$ into $T^{n}$. Since $H^{\prime}$ is dense in $H$, this is impossible. Thus $D$ is finite. Since $H \in[Z]$ (by Theorem 2.1), and $H^{\prime}$ is dense in $H$, it follows from Corollary 1 of Theorem 4.4 that $H$ is compact; hence so is $G$. This completes the proof.

We shall now prove the existence of the periodic subgroup (as defined below) for groups in $[Z]$.

Definition. (1) If $x \in G$, where $G$ is a topological group, then $[x]$ denotes the closure of the subgroup of $G$ generated by $x$. (2) If $[x]$ is compact we say that $x$ is a periodic element (of $G$ ). (3) We denote by $P$ (or $P_{G}$ ) the set of periodic elements of $G$.

THEOREM 5.4. If $G \in[Z]$ then

(1) $P$ is a closed, characteristic subgroup of $G$ containing every compact subgroup;

(2) $P$ consists of those elements $x$ in $G$ which belong to some compact, normal subgroup of $G$;

(3) $G / P$ has no periodic elements (except the identity); in fact, $G / P=W \times D$, where $D$ is a discrete, torsion free, abelian group. 
Proof. (1) By Theorem 4.4, $G=W \times H$, where $H \supseteq K$. Let $x, y \in H$. Let $\pi: H \rightarrow H / K$ denote the canonical epimorphism. Since $[x]$, $[y]$ are compact subgroups of $H$, and since $H / K$ is discrete, $\pi([x])$ and $\pi([y])$ are finite groups. Therefore, $\pi(x), \pi(y)$ are elements of finite order. By Theorem 4.2, the conjugacy classes of elements of groups in $[Z]$ are compact. It follows from this and Theorem 2.1 that $H / K$ is an $F C$-group [14, p. 178]. Hence a theorem of B. Neumann [14, p. 184] shows that $\pi(x y)=\pi(x) \pi(y)$ is of finite order.

Now $\pi([x y]) \subseteq[\pi(x y)]$. In order to verify this, note that $\pi\left((x y)^{n}\right) \in[\pi(x y)]$, for all integers $n$, that $\pi$ is a continuous map, and that $[\pi(x y)]$ is closed. It follows that $[x y] \subseteq \pi^{-1}([\pi(x y)])$. Since $[\pi(x y)]$ is finite (because $H / K$ is discrete) and since $K$ is compact, this implies that $[x y]$ is compact.

Since $\left[x^{-1}\right]=[x]$, this completes the proof that $P$ is a subgroup of $G$. Clearly, $P$ is a characteristic subgroup which contains every compact subgroup of $G$. In particular, $P \supseteq K$, so that $P$ is open in $H$ and therefore closed in $G$.

(2) It suffices to show that if $x \in P$ then $x$ belongs to some compact normal subgroup $K_{x}$ of $G$. As above, $x \in H$ and $\pi(x)$ has finite order. Since $H / K$ is an $F C$-group, it follows from [14, p. 185] that $\pi(x)$ belongs to a finite normal subgroup $K_{\pi(x)}$ of $H / K$. Then $K_{x}=\pi^{-1}\left(K_{\pi(x)}\right)$ is a compact normal subgroup of $H$ containing $x$. Clearly, $K_{x}$ is normal in $G$.

(3) It follows from (1) and from Corollary 1, Theorem 4.4 that $P \supseteq\left(G^{\prime}\right)^{-}$, so that $G / P$ is abelian. Now $G / P \cong W \times(H / P)$ and $H / P$ is discrete. Since $W$ is a vector group, it suffices to show that $H / P$ has no periodic elements. Since this group is discrete, we must show that it is torsion free, i.e., that the following statement holds:

(*) "If $x \in H$ and if $x^{n(x)} \in P$, for some positive integer $n(x)$, then $x \in P$."

This is equivalent to

(**) "If $x \in H$ and $\left[x^{n(x)}\right]$ is compact, for some positive integer $n(x)$, then $[x]$ is compact."

Since $[x]$ is a locally compact abelian group, in order to prove $(* *)$ it is sufficient to prove (**) [i.e., $(*)$ ] for any locally compact abelian group $H$. If $H$ is such a group then, as was noted by Pontrjagin [17, p. 53, vol. 2], $(P, \hat{H})=(\hat{H})_{1}$, where $\hat{H}$ denotes the character group of $H$ and $(P, \hat{H})$ the annihilator of $P$ in $\hat{H}$. Hence $(H / P)^{\wedge}=(\hat{H})_{1}$. Since $(H / P)^{\wedge}$ is connected, $H / P$ is torsion free as can be argued from the abelian theory [12]. Thus $G / P \cong W \times D$, where $D=H / P$ has the requisite properties.

REMARK. If $G$ is an arbitrary locally compact group, $P$ need not be a subgroup, even if $G$ is a Lie group with small invariant neighborhoods of 1 .

COROLlaRY. The real-valued continuous homomorphisms separate the points of $G$ if and only if $G=W \times D$.

Proof. For groups of the form $W \times D$ this is well known. Conversely, any such homomorphism annihilates $P$. Since these functions separate the points of $G, P=(1)$. 
We now investigate under what conditions $P$ is the maximum compact subgroup of $G$, where $G \in[Z]$.

THEOREM 5.5. If $G \in[Z]$ the following conditions are equivalent:

(1) $G$ has a maximum compact subgroup.

(2) $P$ is compact.

(3) $G /\left(G^{\prime}\right)^{-}=W \times L \times D$, where $L$ is compact and $D$ is discrete and torsion free, and $W$ is the vector subgroup of $G$ occurring in the decomposition of $G$ according to Theorem 4.4.

Proof. Let $Q$ be the maximum compact subgroup of $G$; then $Q \subseteq P$, by Theorem 5.4. If $x \in P$ then there exists a compact normal subgroup $K_{x}$ of $G$ containing $x$ (Theorem 5.4). Then $Q K_{x}$ is a compact subgroup of $G$, hence is contained in $Q$. Therefore, $P=Q$.

Now assume that $P$ is compact. Denote by $\omega$ the canonical epimorphism $H \rightarrow H /\left(H^{\prime}\right)^{-}$. Since $\left(H^{\prime}\right)^{-}$is compact (by Corollary 1 of Theorem 4.4), it follows from Theorem 5.4, (2), that $P_{H /\left(H^{\prime}\right)^{-}}=\omega(P)$, so that $P_{H /\left(H^{\prime}\right)^{-}}$is compact. Now it follows from [17, p. 53, Volume 2] that, for any locally compact abelian group $A, P_{A}$ is compact if and only if $(\hat{A})_{1}$ is open in $\hat{A}$. Hence, if we take $A$ to be $H /\left(H^{\prime}\right)^{-}$, it follows that $(\hat{A})_{1}$ is divisible (Corollary to Theorem 5.1 ); furthermore it is open in $\hat{A}$. Consequently, $\hat{A} \cong(\hat{A})_{1} \times\left(\hat{A} /(\hat{A})_{1}\right)$, and hence $A \cong\left(A / P_{A}\right) \times P_{A}$, where $A / P_{A}$ is the direct product of a vector group and a discrete, torsion free group, by Theorem 5.4. Since $H$ contains a compact open subgroup, so does $H /\left(H^{\prime}\right)^{-}$; hence $H /\left(H^{\prime}\right)^{-}$ contains no nontrivial vector subgroups. Hence $H /\left(H^{\prime}\right)^{-} \cong P_{A} \times D$ and therefore $G /\left(G^{\prime}\right)^{-} \cong W \times L \times D$, as asserted.

Assume that (3) holds and note that then $L$ is the maximum compact subgroup of $G /\left(G^{\prime}\right)^{-}$. If $\tau: G \rightarrow G /\left(G^{\prime}\right)^{-}$is the canonical epimorphism then $\tau^{-1}(L)$ is compact. Clearly, it is the maximum compact subgroup of $G$.

COROLlARY 1. If $G$ is a compactly generated group in $[Z]$ then $P$ is the maximum compact subgroup of $G$. Moreover, $G / P \cong W \times Z^{s}$.

Proof. Since $G$ is compactly generated, so is $G /\left(G^{\prime}\right)^{-}$, so that $G /\left(G^{\prime}\right)^{-}$, being a compactly generated abelian group, satisfies (3) of Theorem 5.5. Thus $P$ is the maximum compact subgroup of $G$.

By Theorem $5.4, G \mid P=W \times D$. Since $G$ is compactly generated, $D$ is finitely generated. Hence $D \cong Z^{s}$.

COROLlaRY 2. There is a central subgroup $Z^{r}$ of $G$ such that $G / Z^{r}$ is compact and $r=\operatorname{dim} W+s$.

Proof. Consider the exact sequence

$$
(1) \rightarrow Z(H) \cap P \rightarrow Z(H) \rightarrow Z(H) / Z(H) \cap P \rightarrow(1) .
$$

Since $P$ is compact, we may replace $Z(H) / Z(H) \cap P$ by $P Z(H) / P$. Now $P Z(H) / P$ is a subgroup of $H / P$, which (Corollary 1 ) is isomorphic with $Z^{s}$; hence $P Z(H) / P$ 
is finitely generated. Since $Z(H) \cap P$ is compact (Corollary 1 ) it follows immediately that $Z(H)$ is compactly generated. Consequently, $Z(H)=V \times L \times Z^{t}$, where $L$ is compact. Clearly, $V=(1)$, because $H_{1}=K_{1}$ is compact. Since $H \in[Z], H / Z^{t}$ is compact.

Since $L \subseteq P$, the canonical epimorphism $\omega: H \rightarrow H / P \cong Z^{s}$ annihilates $L$. Hence $\omega(Z(H))=\omega\left(Z^{t}\right)$, and $\omega$ induces a continuous epimorphism $H / Z(H) \rightarrow Z^{s} / \omega\left(Z^{t}\right)$. Since $H \in[Z]$, this implies that the rank of $\omega\left(Z^{t}\right)$ equals s. Since $Z^{t} \cap P=(1)$, it follows that $\omega\left(Z^{t}\right) \cong Z^{t}$. Therefore, $t=s$. Now let $Z^{r}$ be the direct product of a maximal lattice of $W$ with $Z^{t}$. The result follows.

One might conjecture that the group extension obtained in Corollary 1 splits. That this is not the case, even if $G$ is discrete, is shown by the following example $\left({ }^{15}\right)$.

EXAMPLE. Let $Z^{2}$ act trivially on $Z_{2}$, the group of integers modulo 2 . If $a=\left(a_{1}, a_{2}\right)$ and $b=\left(b_{1}, b_{2}\right)$ are points of $Z^{2}$ then the map $f: Z^{2} \times Z^{2} \rightarrow Z_{2}$ defined by $f(a, b)=a_{1}^{*} b_{2}^{*}$ (where * denotes coset modulo 2) defines an element of $H^{2}\left(Z^{2}, Z_{2}\right)$; this cohomology class is nonzero, since there are points $a, b$ such that $f(a, b)$ $\neq f(b, a)$, whereas coboundaries of 1-cochains are symmetric in $a, b$.

Hence if $P=Z_{2}$ and if $G$ is the discrete group consisting of pairs $(p, a), p \in P$, $a \in Z^{2}$, with multiplication defined by $(p, a)(q, b)=(p+q+f(a, b), a+b)$ then the extension (1) $\rightarrow P \rightarrow G \rightarrow G / P \rightarrow(1)$ is not split. On the other hand, $P$ is the torsion subgroup of $G, G$ is finitely generated, and $G \in[Z]$, since $Z(G)$ is of index 4 in $G$.

We note that this situation is markedly different from that of compactly generated abelian groups.

The remainder of this section will be devoted to the study of the division closure of subgroups of groups in $[Z]$, a generalization to topological group theory of a well-known notion of discrete group theory due to P. Alexandroff. To our knowledge, this definition is new; it can be shown to be equivalent to the usual notion of division closure in the case of discrete groups $\left({ }^{17}\right)$.

Definition. (1) Let $G$ be a locally compact topological group, $M$ a (not necessarily closed) subgroup. The division closure of $M$ in $G$, denoted by $(M: G)$, is the set of all elements $x$ of $G$ such that there exists a compact neighborhood $U_{x}$ of 1 in $G$ and $[x] \subseteq U_{x} \bar{M}$. (2) $(M: G)$ is called strongly uniform if there exists a compact neighborhood $U$ of 1 in $G$ such that $[x] \subseteq U \bar{M}$, for all $x$ in $(M: G)$. (3) $M$ is called division closed if $M=(M: G)$.

Remark. We observe that $P_{G}=((1): G)$ and that $(M: G)$ contains $\bar{M}, P_{G}$, and the division closure of $M$ in the sense of discrete groups. If $M$ is compact then $(M: G)=P_{G}$.

THEOREM 5.6. Let $G \in[Z]$ and $M$ be a subgroup of $G$. Then the following holds:

(1) $(M: G)$ is a closed normal subgroup of $G$.

(2) $(M: G)$ is division closed.

${ }^{(17)}$ If $M$ is a subgroup of $G$ then the division closure of $M$ in $G$ is the set of all elements $x$ such that some power of $x$ belongs to $M$. 
(3) $(M: G)=\left((\bar{M} P)^{-}: G\right)$.

(4) $x \in(M: G)$ if and only if there is a closed normal subgroup $Q_{x}$ of $G$ such that $x \in Q_{x}$ and $Q_{x} /(\bar{M} P)^{-}$is compact.

(5) If $M$ is closed and normal, then $(M: G) / M \cong P_{G / M}$. In particular, $M$ is division closed if and only if $G / M$ has no periodic elements.

Proof. (5) Let $x \in(M: G)$. Then $[x] \subseteq U_{x} M$, where $U_{x}$ is as above. Hence, if $\phi: G \rightarrow G / M$ is the canonical epimorphism and $n$ is any integer, then $\phi(x)^{n}$ $=\phi\left(x^{n}\right) \in \phi\left(U_{x}\right)$, a compact neighborhood of 1 in $G / M$. Thus $[\phi(x)]$ is compact, and therefore $\phi(x) \in P_{G / M}$. On the other hand, if, for some $x$ in $G,[\phi(x)]$ is compact then, for every integer $n, \phi\left(x^{n}\right) \in U_{x}^{*}$, a compact neighborhood of 1 in $G / M$. Since $G$ is locally compact, there exists a compact neighborhood $U_{x}$ of 1 in $G$ such that $\phi\left(U_{x}\right) \supseteq U_{x}^{*}$. Hence $x^{n} \in U_{x} M$, and therefore $x \in(M: G)$. Since $G / M \in[Z], P_{G / M}$ is a closed normal subgroup of $G / M$ (Theorem 5.4), so that $(M: G)$ is, in fact, a closed normal subgroup of $G$. The remaining assertions of (5) follow.

(1) By Corollary 1 , Theorem $4.4,\left(G^{\prime}\right)^{-}$is compact. Using this fact one sees immediately from the definitions that $(M: G)=\left(\left(M G^{\prime}\right)^{-}: G\right)$. Now $\left(M G^{\prime}\right)^{-}$is a closed subgroup of $G$; it is also normal, because it contains $G^{\prime}$. Hence for the purpose of proving (1) we may assume that $M$ is closed and normal. However, as was shown in (5), in this case, $(M: G)$ is a closed normal subgroup of $G$.

(2) As in the proof of (1), we may assume that $M$ is closed and normal. Let $x \in(D: G)$, where $D=(M: G)$, and let $\phi: G \rightarrow G / M$ be the canonical epimorphism. From the fact that $D$ is closed in $G$ (by (1)) and the fact that $P_{G / M}=\phi(D)$ (by (5)) it follows that $\phi(x) \in\left(P_{G / M}: G / M\right)$. Since $G / M \in[Z],(G / M) / P_{G / M}$ has no periodic elements (Theorem 5.4) so that, by (5), $P_{G / M}$ equals its division closure, and $\phi(x) \in P_{G / M}$. Hence $x \in D$.

(3) Since $(M: G)$ is a closed subgroup of $G$ (by (1)) containing $\bar{M}$ and $P$, it contains $(\bar{M} P)^{-}$. Since it is also division closed (by (2)) it contains $\left((\bar{M} P)^{-}: G\right)$.

(4) Let $x \in(M: G)$, i.e., $x \in\left((\bar{M} P)^{-}: G\right)$. By Theorem 5.4, $P \supseteq\left(G^{\prime}\right)^{-}$. Hence $(\bar{M} P)^{-}$is normal in $G$. If $\phi: G \rightarrow G /(\bar{M} P)^{-}$denotes the canonical epimorphism, then $\phi(x)$ is a periodic element of $G /(\bar{M} P)^{-}$(by (5)). Hence, by Theorem 5.4, (2), $\phi(x) \in K_{x}$, where $K_{x}$ is a compact normal subgroup of $G /(\bar{M} P)^{-}$. Then $Q_{x}=\phi^{-1}\left(K_{x}\right)$ satisfies the required conditions. The converse is easy to prove.

COROLlARY 1. If $G$ is a nontrivial group in [Z] then there is a closed normal subgroup $M$ of $G$ such that $P_{G / M} \neq(1)$.

Proof. Suppose $P_{G / M}=(1)$, for every closed normal subgroup $M$ of $G$. Then, by Theorem 5.6, (5), every such $M$ is division closed. Hence, in particular, $P=(1)$, and $G=V \times D$, where $D$ is a torsion free, discrete, abelian group (Theorem 5.4). If $\operatorname{dim} V>0$, let $M$ be the direct product of the set of lattice points of $V$ with $D$. Clearly, $(M: G)=G$. Therefore, $V=(1)$ and $G=D$. Now, for each positive integer $n$, the subgroup $n D$ has the property that $D / n D$ is of finite order. Hence $(n D: D)$ $=D$, so that $n D=D$, and $D$ is divisible. As a divisible, torsion free, abelian group, 
$D$ is a weak direct sum of groups of rational numbers. Taking the quotient of this modulo the corresponding weak direct sum of integers, we get a contradiction to the condition $P_{G / M}=(1)$, unless $D=(1)$.

Corollary 2. Let $G \in[Z]$ and $M$ be a closed normal subgroup of $G$. Then $(M: G)$ is strongly uniform if and only if $G / M$ has a maximum compact subgroup.

Proof. If $(M: G)$ is strongly uniform, then there is a compact neighborhood $U$ of 1 in $G$ such that $(M: G) \subseteq U M$. Hence $(M: G) / M$ is compact. By Theorem 5.6, (5), this equals $P_{G / M}$, so that, by Theorem 5.5, $G / M$ has a maximum compact subgroup. One proves the converse by reversing the above conclusions and using the fact that $G$ is locally compact.

We conclude $\$ 5$ with two remarks on the lattice theoretic properties of the division closure.

Remark 1. It follows from the definitions and from (1), (2) of Theorem 5.6 that the set of division closed subgroups of a group $G$ in $[Z]$ is a complete lattice in the sense of lattice theory.

REMARK 2. If $S$ is an arbitrary subset of $G$, where $G \in[Z]$, define $(S: G)$ to be $(M: G)$, where $M$ is the subgroup of $G$ generated by $S$. It then follows from the above that the operation $S \rightarrow(S: G)$ is a closure operation, in the sense of lattice theory, on the power set of $G$.

\section{REFERENCES}

1. P. Cartier, Séminaire "Sophus Lie," Ecole Norm. Sup., Paris, 1954/1955; Faculté Sci. Paris, Paris, 1955/1956.

2. V. M. Gluškov, The structure of locally compact groups and Hilbert's fifth problem, Amer. Math. Soc. Transl. (2) 15 (1960), 55-93.

3. R. Godement, Analyse harmonique dans les groupes centraux. I. Fonctions centrales et caractères, C. R. Acad. Sci. Paris 225 (1947), 19-21.

4. - Review of F. I. Mautner, Infinite-dimensional irreducible representations of certain groups, Math. Reviews 12 (1951), p. 588.

5. G. Hochschild, The structure of Lie groups, Holden-Day, San Francisco, Calif., 1965.

6. K. H. Hofmann and Paul Mostert, Splitting in topological groups, Mem. Amer. Math. Soc. No. 43 (1963), 75 pp.

7. K. Iwasawa, Topological groups with invariant compact neighborhoods of the identity, Ann. of Math. 54 (1951), 345-348.

8. E. van Kampen, Locally bicompact abelian groups and their character groups, Ann. of Math. 36 (1935), 448-463.

9. J. L. Kelley, General topology, Van Nostrand, Princeton, N. J., 1952.

10. A. Mal'cev, On the simple connectedness of invariant subgroups of Lie groups, C. R. (Doklady) Acad. Sci. URSS (N.S.) 34 (1942), 10-13.

11. D. Montgomery and L. Zippin, Topological transformation groups, Interscience, New York, 1955.

12. M. Moskowitz, Homological algebra in locally compact abelian groups, Trans. Amer. Math. Soc. 127 (1967), (to appear).

13. G. D. Mostow, On an assertion of Weil, Ann. of Math. 54 (1951), 339-344. 
14. B. H. Neumann, Groups with finite classes of conjugate elements, Proc. London Math. Soc. 1 (1951), 178-187.

15. - Groups with finite classes of conjugate subgroups, Math. Z. 63 (1955), 76-96.

16. L. Pontrjagin, Topological groups, Princeton Univ. Press, Princeton, N. J., 1939.

17. —, Topologische Gruppen, Teil I, II, Teubner, Leipzig, 1957.

18. P. Smith, On the fundamental group of a group manifold, Ann. of Math. 36 (1935), 210-229.

19. A. Weil, L'intégration dans les groupes topologiques et ses applications, Hermann, Paris, 1953.

\author{
UNIVERSTTY OF CALIFORNIA, \\ Berkeley, California \\ CoRnell University, \\ ITHACA, New YoRK \\ UNIVERSITY OF Chicago, \\ Chicago, Illinois
}

